\title{
In silico drug repurposing for SARS-CoV-2 Main Proteinase and Spike proteins
}

\author{
Irene Maffucci ${ }^{1, *}$, Alessandro Contini ${ }^{2, *}$ \\ ${ }^{1}$ Université de technologie de Compiègne, UPJV, CNRS, Enzyme and Cell Engineering, Centre \\ de recherche Royallieu - CS 60319 - 60203 Compiègne Cedex \\ ${ }^{2}$ Università degli Studi di Milano, Dipartimento di Scienze Farmaceutiche, Sezione di Chimica \\ Generale e Organica “A. Marchesini”, Via Venezian, 2120133 Milano, Italy
}




\section{Index}

Figure S1. RMSD of the RBD-hACE2 complex backbone atoms from the X-ray structure (code PDB 6M0J) during the $20 \mathrm{~ns}$ MD simulation. S3

Table S1. Difference in the binding free energies between the mutated and the native RBD-hACE2 complex obtained from the alanine scanning performed on the last $10 \mathrm{~ns}$ of the MD simulation of the complex by individually mutating the RBD residues at the interface with hACE2. The numbering has been kept in according to the X-ray structure. S3

Table S2. Compounds selected by docking on $\mathrm{M}^{\text {pro }}$ (6LU7 model) that failed during the MD/NwatMMGBSA rescoring step.

Table S3. Compounds selected by docking on $\mathrm{M}^{\text {pro }}$ (QHD43415 homology model) that failed

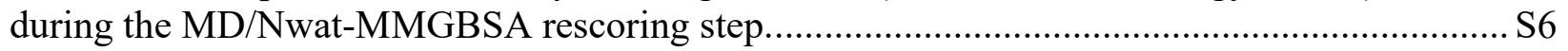

Table S4. Hydrogen bonds between RBD and hACE2 during the last half of the 20 ns MD simulation. Cut-offs of $3.5 \AA$ and $150^{\circ}$ have been used. The average donor-acceptor distances (AvgDist) are reported in $\AA$ and the average donor-donorH-acceptor angles are reported in degrees.

Figure S2. Superposition of thymopentin docked to RBD BS2 (light green) and the PEPFOLD3 structure prediction (dark green). The backbone RMSD between the two structures is $1.3 \AA$.... S 8 


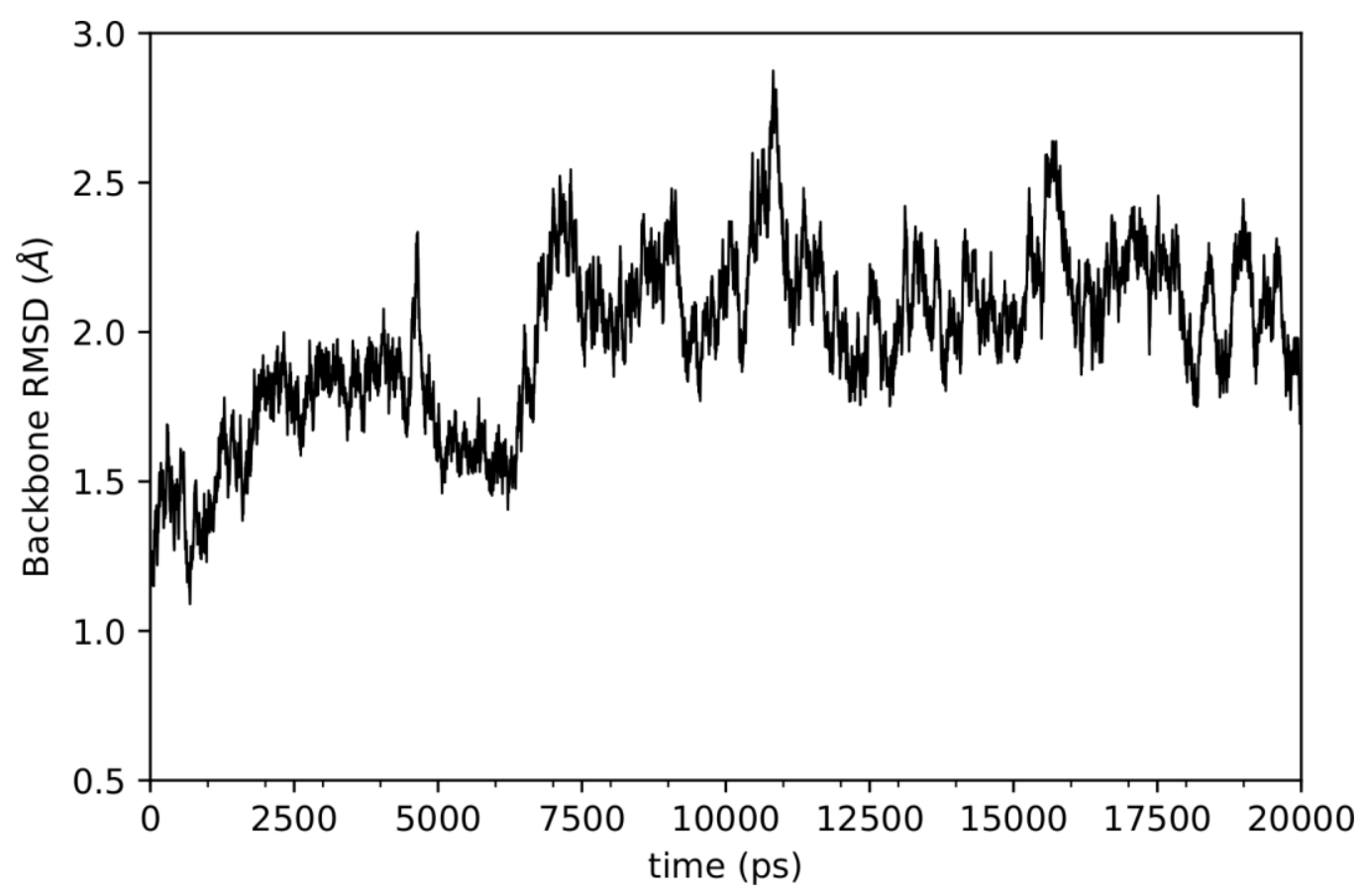

Figure S1. RMSD of the RBD-hACE2 complex backbone atoms from the X-ray structure (code PDB 6M0J) during the $20 \mathrm{~ns}$ MD simulation.

Table S1. Difference in the binding free energies between the mutated and the native RBD-hACE2 complex obtained from the alanine scanning performed on the last $10 \mathrm{~ns}$ of the MD simulation of the complex by individually mutating the RBD residues at the interface with hACE2. The numbering has been kept in according to the X-ray structure.

\begin{tabular}{|l|l|l|}
\hline \#Residue & $\boldsymbol{\Delta} \mathbf{\Delta G}(\mathbf{k c a l} / \mathbf{m o l})$ & Standard deviation $(\mathbf{k c a l} / \mathbf{m o l})$ \\
\hline R403 & 0.22 & 0.25 \\
\hline K417 & 2.23 & 2.17 \\
\hline Y449 & 2.74 & 1.35 \\
\hline Y453 & 0.10 & 0.60 \\
\hline L455 & 3.00 & 0.73 \\
\hline F456 & 3.57 & 1.06 \\
\hline
\end{tabular}




\begin{tabular}{|l|l|l|}
\hline Y473 & 0.40 & 0.24 \\
\hline S477 & 0.22 & 1.55 \\
\hline T478 & -0.07 & 0.10 \\
\hline E484 & -0.11 & 0.12 \\
\hline F486 & 4.45 & 1.55 \\
\hline N487 & 4.02 & 1.49 \\
\hline Y489 & 3.29 & 0.72 \\
\hline F490 & -0.09 & 0.05 \\
\hline L492 & 0.07 & 0.03 \\
\hline Q493 & 4.75 & 1.89 \\
\hline S494 & 0.06 & 0.08 \\
\hline Y495 & -0.08 & 0.05 \\
\hline Q498 & 9.13 & 3.43 \\
\hline T500 & 2.58 & 1.27 \\
\hline N501 & 4.25 & 1.44 \\
\hline V503 & -0.01 & 0.09 \\
\hline Y505 & 4.64 & 1.81 \\
\hline
\end{tabular}

Table S2. Compounds selected by docking on $\mathrm{M}^{\mathrm{pro}}$ (6LU7 model) that failed during the MD/NwatMMGBSA rescoring step.

\begin{tabular}{lll}
\hline Drug Name & Dock score & MD/Nwat-MMGBSA \\
\hline Nafarelin & -155.4 & $\mathrm{~F}$ \\
Leuprorelin & -145.6 & $\mathrm{~F}$ \\
Leuprorelin & -140.9 & $\mathrm{~F}$ \\
Somatostatin & -139.6 & $\mathrm{~F}$ \\
Icatibant & -139.3 & $\mathrm{~F}$ \\
\hline
\end{tabular}




\begin{tabular}{lll}
\hline Nystatin & -132.7 & $\mathrm{~F}$ \\
Goserelin & -128.8 & $\mathrm{~F}$ \\
Alarelin & -128.5 & $\mathrm{~F}$ \\
Gonadorelin & -125.5 & $\mathrm{~F}$ \\
Amphotericin B & -124.7 & $\mathrm{~F}$ \\
Carfilzomib & -122.4 & $\mathrm{~F}$ \\
Thymopentin & -121.2 & $\mathrm{~F}$ \\
Lentinan & -116.4 & $\mathrm{~F}$ \\
Ritonavir & -116.1 & $\mathrm{~F}$ \\
NAD+ & -114.1 & $\mathrm{~F}$ \\
Octreotide & -113.6 & $\mathrm{~F}$ \\
Colistin & -113.5 & $\mathrm{~F}$ \\
Cangrelor & -111.6 & $\mathrm{~F}$ \\
Oxytocin & -111.4 & $\mathrm{~F}$ \\
Flucytosine & -111.3 & $\mathrm{~F}$ \\
Echinacoside & -110.3 & $\mathrm{~F}$ \\
Monomethyl auristatin E & -110.2 & $\mathrm{~F}$ \\
Salmeterol & -110.1 & $\mathrm{~F}$ \\
Daptomycin & -109.9 & $\mathrm{~F}$ \\
Pneumocandin B0 & -109.6 & $\mathrm{~F}$ \\
Caspofungin & -109.1 & $\mathrm{~F}$ \\
Vilanterol & -109.1 & $\mathrm{~F}$ \\
Stachyose & -108.2 & $\mathrm{~F}$ \\
Ginsenoside Rb1 & -108.1 & $\mathrm{~F}$ \\
Venetoclax & -107.7 & $\mathrm{~F}$ \\
Terlipressin & -106.7 & $\mathrm{~F}$ \\
\hline
\end{tabular}




\begin{tabular}{lll}
\hline Atracurium besylate & -106.5 & F \\
Polymyxin B & -106.5 & F \\
Deferoxamine mesylate & -106.2 & F \\
Darunavir & -105.9 & F \\
\hline
\end{tabular}

Table S3. Compounds selected by docking on $\mathrm{M}^{\text {pro }}$ (QHD43415 homology model) that failed during the MD/Nwat-MMGBSA rescoring step.

\begin{tabular}{lll}
\hline Drug Name & Dock score & MD/Nwat-MMGBSA \\
\hline Gonadorelin & -131.4 & $\mathrm{~F}$ \\
Leuprorelin & -127.9 & $\mathrm{~F}$ \\
Nafarelin & -127.9 & $\mathrm{~F}$ \\
Leuprorelin & -124.1 & $\mathrm{~F}$ \\
Goserelin & -117.8 & $\mathrm{~F}$ \\
Bacitracin & -117.0 & $\mathrm{~F}$ \\
Amphotericin B & -115.2 & $\mathrm{~F}$ \\
Alarelin & -114.6 & $\mathrm{~F}$ \\
Deferoxamine & -113.7 & $\mathrm{~F}$ \\
Nystatin & -112.8 & $\mathrm{~F}$ \\
Octreotide & -108.0 & $\mathrm{~F}$ \\
Carfilzomib & -107.7 & $\mathrm{~F}$ \\
Terlipressin & -107.3 & $\mathrm{~F}$ \\
Somatostatin & -106.5 & $\mathrm{~F}$ \\
Flucytosine & -106.4 & $\mathrm{~F}$ \\
Lypressin & -106.3 & $\mathrm{~F}$ \\
Sennoside A & -105.6 & $\mathrm{~F}$ \\
Colistin & -103.9 & $\mathrm{~F}$ \\
\hline
\end{tabular}


Polymyxin B

Nelfinavir

Madecassoside

Asiaticoside

Pneumocandin B0

Stevioside

Thymopentin

Tenofovir Disoproxil Fumarate

Lapatinib

Octreotide

Desmopressin

NAD+

$\begin{array}{ll}-103.3 & F \\ -102.7 & F \\ -102.5 & F \\ -102.4 & F \\ -102.4 & F \\ -101.4 & F \\ -100.4 & F \\ -100.3 & F \\ -99.3 & F \\ -99.2 & F \\ -99.1 & F \\ -98.5 & F\end{array}$

Table S4. Hydrogen bonds between RBD and hACE2 during the last half of the $20 \mathrm{~ns}$ MD simulation. Cut-offs of $3.5 \AA$ and $150^{\circ}$ have been used. The average donor-acceptor distances (AvgDist) are reported in $\AA$ and the average donor-donorH-acceptor angles are reported in degrees.

\begin{tabular}{|l|l|l|l|l|l|}
\hline \#Acceptor & DonorH & Donor & Frac & AvgDist & AvgAng \\
\hline LYS_353@O & GLY_502@H & GLY_502@N & 0.9026 & 2.8952 & 164.4105 \\
\hline ASN_487@OD1 & TYR_83@HH & TYR_83@OH & 0.6796 & 2.7777 & 162.7078 \\
\hline ASP_355@OD2 & THR_500@HG1 & THR_500@OG1 & 0.5978 & 2.7536 & 163.9769 \\
\hline GLU_35@OE2 & GLN_493@HE22 & GLN_493@NE2 & 0.5772 & 2.88 & 164.7713 \\
\hline ASP_38@OD1 & TYR_449@HH & TYR_449@OH & 0.4672 & 2.721 & 164.7831 \\
\hline ASP_38@OD2 & TYR_449@HH & TYR_449@OH & 0.4454 & 2.7187 & 164.3915 \\
\hline ASP_38@OD2 & GLN_498@HE22 & GLN_498@NE2 & 0.4424 & 2.9078 & 164.7138 \\
\hline ASP_38@OD1 & GLN_498@HE22 & GLN_498@NE2 & 0.3782 & 2.8983 & 164.6202 \\
\hline
\end{tabular}




\begin{tabular}{|l|l|l|l|l|l|}
\hline GLU_37@OE2 & TYR_505@HH & TYR_505@OH & 0.3434 & 2.7076 & 163.8788 \\
\hline GLU_35@OE1 & GLN_493@HE22 & GLN_493@NE2 & 0.2426 & 2.8978 & 164.91 \\
\hline TYR_41@OH & THR_500@HG1 & THR_500@OG1 & 0.2272 & 2.8517 & 163.2088 \\
\hline ASP_30@OD2 & LYS_417@HZ3 & LYS_417@NZ & 0.218 & 2.774 & 162.3672 \\
\hline ASP_30@OD2 & LYS_417@HZ1 & LYS_417@NZ & 0.2116 & 2.7782 & 161.6638 \\
\hline GLN_498@OE1 & LYS_353@HZ3 & LYS_353@NZ & 0.1462 & 2.8426 & 161.4818 \\
\hline GLN_498@OE1 & LYS_353@HZ2 & LYS_353@NZ & 0.1402 & 2.8527 & 161.641 \\
\hline ASP_30@OD2 & LYS_417@HZ2 & LYS_417@NZ & 0.1324 & 2.7924 & 162.3512 \\
\hline GLN_498@OE1 & LYS_353@HZ1 & LYS_353@NZ & 0.1298 & 2.8405 & 161.1518 \\
\hline GLN_493@OE1 & LSY_31@HZ1 & LSY_31@NZ & 0.1262 & 2.8247 & 162.1882 \\
\hline GLN_493@OE1 & LSY_31@HZ3 & LSY_31@NZ & 0.1252 & 2.8188 & 161.9295 \\
\hline GLU_37@OE1 & TYR_505@HH & TYR_505@OH & 0.1176 & 2.7326 & 163.3618 \\
\hline GLN_493@OE1 & LSY_31@HZ2 & LSY_31@NZ & 0.1168 & 2.8314 & 162.1823 \\
\hline GLN_24@OE1 & ASN_487@HD21 & ASN_487@ND2 & 0.1118 & 2.9744 & 159.9545 \\
\hline GLN_24@OE1 & SER_477@H & SER_477@N & 0.1114 & 2.9675 & 163.4158 \\
\hline
\end{tabular}

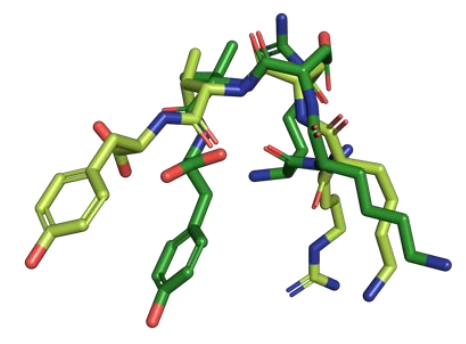

Figure S2. Superposition of thymopentin docked to RBD BS2 (light green) and the PEPFOLD3 structure prediction (dark green). The backbone RMSD between the two structures is $1.3 \AA$. 